\title{
Photosynthetic Algae-Insulinoma Cell Fusion Creating Self-Sustaining Insulin Producer
}

\author{
D. Heller ${ }^{1}$, A. Calabro ${ }^{2}$, C. Queenan ${ }^{2}$, R. Pergolizzi ${ }^{1}$ \\ 1. Bergen County Academies, Cell Biology Lab, 200 Hackensack Avenue, Hackensack, NJ 07601 \\ 2. Bergen County Academies, Nano-Structural Imaging Lab, 200 Hackensack Avenue, Hackensack, NJ \\ 07601
}

Insulin is a pancreatic hormone that regulates blood sugar concentration. In the approximately 300 million diabetic patients worldwide, utilization and production of insulin is substantially, and in the case of Diabetes mellitus type I patients, completely, impaired [1]. These patients require direct pumping of exogenous insulin into the blood. There are currently two methods of exogenous insulin production: extraction from other genetically similar mammals (such as bovine), or transfection of bacteria with the human genes that code for insulin protein production. The first method has become increasingly uncommon due to allergic reactions to insulin produced by animals. The second is slightly inefficient only in that it requires the use of complex protocols in order to generate sufficient quantities of insulin [2].

This study seeks to create a new method for efficient in vitro production of exogenous insulin. The basis behind the method is the creation of a low maintenance, self-sustaining cell hybrid that produces insulin, and energy via photosynthesis. The photosynthetic green-algae species, Chlorella kessleri, and rat insulinoma cell line, $R I N-5 F$, were fused to create cell hybrid cells referred to as Modified Insulin Production (MIP) cells.

It was hypothesized that successful fusion of algae and insulinomas would lead to an efficient, inexpensive approach to in vitro insulin production via plant-animal cell hybrids containing the biochemical properties of each cell type. Prior to fusion, the rigid cell walls of the algae cells were removed by the cell wall-degrading enzymes, cellulase and pectinase, creating algal protoplasts [3]. Protoplast isolation is essential, as the rigid cell walls restrict cell wall interaction with the insulinomas. Fusion was then stimulated with polyethylene glycol (PEG, MW 4000) [4]. Ultra-Sensitive Rat Insulin ELISA was performed on the pre-fused insulinomas and on the MIP cell hybrids to quantify insulin secretion in media. It was found that the fusions produced approximately $40 \mathrm{ng} / \mathrm{mL}$ insulin in media. The expensive DMEM/F12 media used initially for the fusions was diluted with a cheap media of salts (ATCC Media 5), yeast, and beef extract. The fusions remained viable and produced insulin for 14 days after dilution with the less expensive media.

Algae cells were imaged by transmission electron microscopy prior to fusion and successful fusion was observed by light microscopy. The algae-insulinoma fusion process represents the potential for the inexpensive production of life-saving bioproducts by means of multi-faceted cell hybrids. Theoretically, any cell type can be substituted in place of the insulinomas (i.e. pituitary gland cells, adrenal gland cells) during the fusion process. The photosynthetic (and glucose producing) properties of the algae act as a base upon which any cell type can remain viable and functioning, producing its specific bioproduct without the need for intensive procedures.

References:

[1] A. Thayer, Chemistry and Engineering News (2011). 
[2] A. Riggs, Diabetes Care, Vol. 4, No.1, (1981).

[3] L. Yang, et al., Cellulose (2008) 789-796.

[4] Y. Lee, et al., J. General Microbiology (1987) 635-641.

[5] The authors would like to acknowledge the administration of the Bergen County Technical Schools for their continued support of the research program.

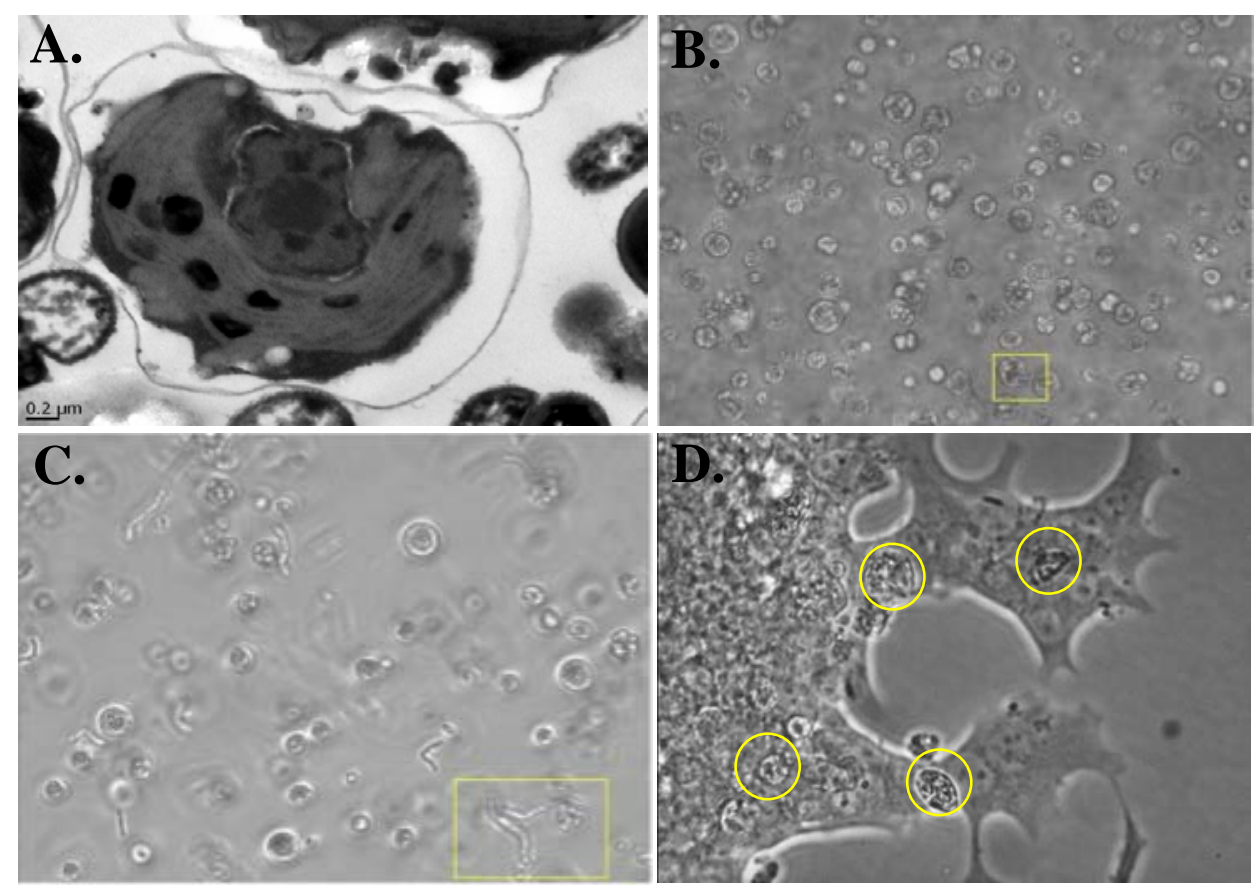

Figure 1: A) Transmission electron micrograph of C. keslerri; notice thick cell wall surrounding chloroplast-filled cytoplasm. B) Light micrograph of C. keslerri before degradation of cell wall. C) Light micrograph of C. keslerri after degradation of cell wall D) Light micrograph of MIP cell hybrid between $C$. keslerri (circled) and RIN-5F.

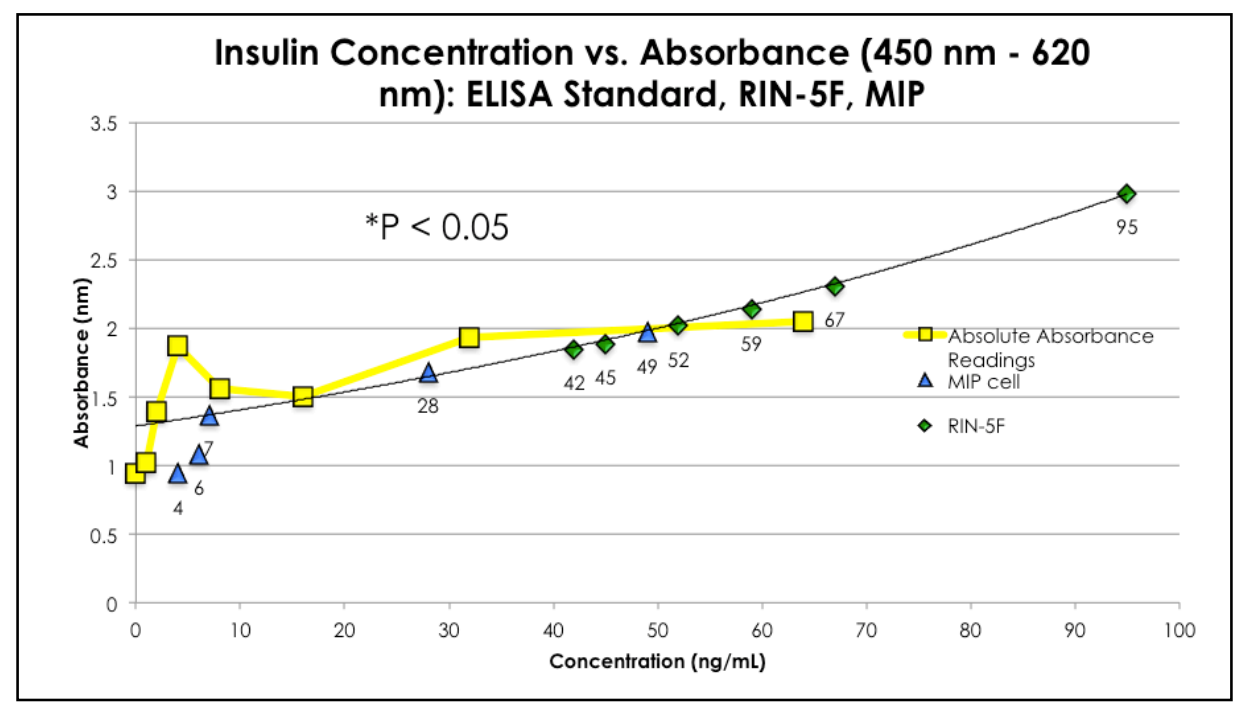

Figure 2: Insulin ELISA results. Concentrations were determined from standard curve (black curved line). Statistical significance was determined between standard insulin readings, MIP cell readings, and RIN-5F results. 